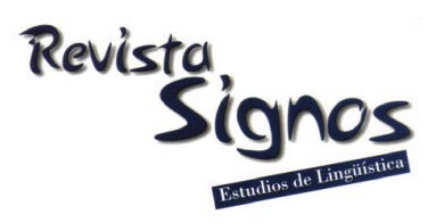

\title{
Enseñar a escribir un artículo de investigación mediante la revisión colaborativa: Percepciones de los estudiantes
}

\section{Teaching to write an academic research paper through collaborative revision: The students' perceptions}

\author{
Mariona Corcelles \\ UNIVERSITAT RAMON LLULL \\ EsPAÑA \\ marionacs@blanquerna.url.edu
}

\author{
Maribel Cano \\ UNIVERSITAT RAMON LLULL \\ ESPAÑA \\ maribelco@blanquerna.url.edu

\section{Montserrat Castelló \\ UNIVERSITAT RAMON LLULL \\ ESPAÑA \\ montserratcb@blanquerna.url.edu}

Recibido: 17-VII-2015 / Aceptado: 24-II-2017

\section{Resumen}

Escribir un artículo académico de investigación (AAI) en el contexto de la tesis final de grado (TFG), puede resultar muy difícil sin la ayuda de una intervención específicamente diseñada para este propósito. En este estudio describimos una propuesta de enseñanza de la escritura académico-científica basada en la revisión colaborativa y analizamos las percepciones de los estudiantes sobre su proceso de escritura y sobre la intervención realizada. Se administró una encuesta durante y después de la escritura del artículo de investigación a 48 estudiantes de $4^{\circ}$ grado de educación de una universidad española. Los resultados muestran que la mayoría de los estudiantes percibieron la escritura de un artículo de investigación como una herramienta para participar en su comunidad profesional y desarrollar su rol docenteinvestigador, aunque también revelaron que se trataba de una actividad compleja cuyo desarrollo generó sentimientos tanto de satisfacción como de ansiedad. El desconocimiento del género AAI, del proceso de investigación, y la gestión del tiempo fueron percibidos como las principales dificultades. Desde la perspectiva de los estudiantes, la revisión colaborativa y las ayudas proporcionadas durante la intervención educativa contribuyeron a superar estas dificultades y al aprendizaje de la escritura académico-científica.

Palabras Clave: Tesis final de grado, escritura académico-científica, artículo académico de investigación, enseñanza de la escritura, revisión colaborativa, educación superior. 


\begin{abstract}
Writing an academic research paper (ARP) based on the undergraduate thesis (UT) can be very difficult without an educational intervention specifically designed for this purpose. In this study, we describe a proposal of teaching academic research writing based on collaborative peer review and analyze students' perceptions of their writing process and the writing intervention itself. One survey was administered during and after the ARP writing to 48 students in the fourth grade of teaching education. Results showed that most of the students perceived writing an ARP as a tool to engage in their professional community and to develop their role as teacher-researcher, although it was also considered as a complex activity whose development led to feelings of satisfaction as well as anxiety. The lack of awareness about the ARP, the research process as well as time management were perceived as the main difficulties. From the students' perceptions, the collaborative peer review and the assistance provided during the educational intervention contributed to overcome difficulties and promoted their learning of academic research writing.
\end{abstract}

Key Words: Undergraduate thesis, academic research writing, academic research article, teaching writing, collaborative peer review, higher education.

\title{
INTRODUCCIÓN
}

En los últimos años, el desarrollo de la competencia investigadora de los estudiantes universitarios ha ido gradualmente ganando relevancia en los curricula universitarios. Concretamente, la reforma impulsada por la Creación del Espacio Europeo de Educación Superior (EEES) ha conllevado la introducción de materias relacionadas con la investigación, como la Tesis Final de Grado ${ }^{1}$ (a partir de ahora TFG) que requiere el dominio de la lectura y la escritura académico-científica.

Actualmente, la formación de los futuros maestros tiene el reto de formar a los docentes como profesionales reflexivos, que investigan para interpretar y mejorar su ejercicio profesional (Giroux, 1990; Schön, 2002) y que no solo se erigen como consumidores de los resultados de la investigación (Beijaard, Verloop \& Vermunt, 2000), algo que también se observa en otras disciplinas (Aguayo, Castelló \& Monereo, 2014). Se trata pues de propiciar una enseñanza orientada a mejorar la formación científica, a aprender a utilizar la escritura para indagar, innovar y compartir con la comunidad disciplinar un conocimiento realmente capaz de transformar la práctica educativa (Zeichner, 1993; Latorre, 2003; Zeichner \& Liston, 2014).

Aun cuando esta formación científica de los educadores, con grados y matices diversos, debería estar presente en los distintos cursos y materias del grado, la realización de la TFG ofrece una magnífica oportunidad para desarrollar parte de esta formación mediante la práctica de la escritura académico-científica. Escribir un artículo de investigación no solo requiere la activación de capacidades complejas como son la selección, síntesis y análisis crítico de la información, el uso de técnicas para la recolección y el análisis de los datos, sino que también exige aprender a comunicar las ideas empleando los recursos, convenciones y el lenguaje propio de una 
determinada comunidad disciplinar y/o profesional, lo cual puede resultar muy difícil sin la ayuda de una intervención específicamente diseñada para este propósito. Éste es, precisamente, el foco de nuestro estudio en el que presentamos una propuesta de enseñanza de la escritura académico-científica basada en la revisión colaborativa que pretende facilitar este aprendizaje. A partir de su implementación los objetivos específicos del estudio fueron: A) analizar las percepciones, sentimientos y dificultades asociadas al proceso de escritura de un artículo de investigación, y B) analizar las percepciones de los estudiantes sobre el aprendizaje realizado y las ayudas recibidas durante la intervención educativa.

\section{Marco teórico}

La tesis final de grado representa el trabajo autónomo de investigación más importante que realiza el estudiante a lo largo de todo el grado. En nuestro contexto su escritura requiere la elaboración de un artículo de investigación, lo que sitúa a los estudiantes ante un género complejo que ha sido denominado 'académico-científico' (Castelló \& Iñesta, 2012), dado que se sitúa a medio camino entre los géneros académicos y los científicos: por un lado, implica escribir un texto que va a ser leído y evaluado por uno -o varios- profesores, lo que le confiere su carácter académico; pero por otro lado, al tratarse de un artículo de investigación, también requiere que el estudiante se posicione como un investigador capaz de producir un texto susceptible de ser publicado y leído por los miembros de la comunidad científica de referencia. Esta doble finalidad acostumbra a generar tensiones en los estudiantes que transitan entre su actual y ya conocido rol como estudiantes y el nuevo rol de investigadores que deben adoptar para escribir un artículo de investigación (Castelló \& Iñesta, 2012; Russell \& Cortes, 2012).

Distintos estudios han mostrado las bondades de realizar una TFG, independientemente del género en el que se inscriba el texto final requerido. Así, aquellos que se han ocupado de analizar las percepciones de los estudiantes indican que la mayoría perciben esta actividad como una experiencia positiva y enriquecedora a nivel personal y profesional. Los estudiantes consideran que se trata de una oportunidad para aprender a pensar y trabajar como científicos, o desarrollar competencias como la del pensamiento crítico (Ryder, 2004; Seymour, Hunter, Laursen \& DeAntoni, 2004). Además, en el ámbito de la formación de maestros la posibilidad de integrar la TFG con el trabajo desarrollado en el practicum puede ser una buena oportunidad para acercar la investigación a las escuelas y permitir a los estudiantes implicarse en proyectos de innovación educativa que respondan a las necesidades y preocupaciones actuales (Pañellas, Aguacil, Boqué \& Rosich, 2013).

La investigación ha puesto de manifiesto que los estudiantes consideran clave por un lado, la elección de un tema de investigación adecuado, ya que éste garantizará en 
gran parte su motivación a lo largo de todo el proceso y la posibilidad de disfrutar del trabajo autónomo (Todd, Bannister \& Clegg, 2004; Harland, Pitt \& Saunders, 2005; Harrison \& Whalley, 2006, 2008). Por otro lado, destacan la importancia de una buena relación con su director, así como la calidad y la frecuencia del feedback que reciben (Todd et al., 2004; Harrison \& Whalley, 2006, 2008; Heinze \& Heinze, 2009; Derounian, 2011). Finalmente, las ayudas entre iguales mediante grupos, tutorías o revisiones de texto colaborativas también han demostrado ser una herramienta eficaz para facilitar el acompañamiento de los estudiantes a lo largo de su proceso de escritura y de investigación (DiPardo \& Freedman, 1988; Heylings \& Tariq, 2001; Inman \& Silverstein, 2003; Orsmond, Merry \& Reiling, 2004; Vargas Franco, 2005; Castelló, Iñesta, Pardo, Liesa \& Martínez-Fernández, 2012; Corcelles, Cano, Bañales \& Vega, 2013).

Sin embargo, distintos estudios ponen de manifiesto que los estudiantes perciben dificultades cuando enfrentan este tipo de trabajos. Les preocupa la gestión del tiempo y del estrés, la falta de habilidades para la investigación, y especialmente la falta conocimientos y habilidades relativas al dominio de las convenciones propias de la escritura académico-científica (Todd et al., 2004; Harrison \& Whalley, 2006, 2008; Castelló \& Iñesta, 2012; Russell \& Cortes, 2012; López-Cózar, Priede \& Benito, 2013).

En el momento de escribir un artículo de investigación, muchos estudiantes se enfrentan por primera vez a la lectura y escritura de textos cuyos destinatarios son habitualmente otros investigadores o profesionales de la comunidad disciplinar, lo que les exige una aproximación diferente (Carlino, 2003, 2005; Solé \& Miras, 2007). Los estudios que analizan las características y finalidades de este género muestran que en un artículo de investigación se espera que los estudiantes se posicionen como autores, es decir hagan visible su 'voz', y elaboren un discurso argumentativo para contrastar sus ideas con las 'voces' de otros autores cercanos a su contexto disciplinar; así es como se elabora el conocimiento que surge de este esfuerzo colectivo para incrementar, profundizar y transformar las ideas de la comunidad científica (Kruse, 2003; Castelló, Corcelles, Iñesta, Vega \& Bañales, 2011; Rinck \& Boch, 2012). Así pues, los estudiantes deben aprender a usar la escritura no solo para describir el conocimiento, sino para desarrollar sus ideas y construir nuevo conocimiento de tal manera que la propia escritura se convierta en una herramienta para reflexionar y apropiarse de los discursos de la comunidad disciplinar (Bazerman, Little, Chavkin, Fouquette, Bethel \& Garufis, 2005; Carlino, 2003). El conocimiento de las particularidades del género y de los recursos propios de la comunidad disciplinar -a la que se dirige el estudiante cuando escribe un artículo de investigación- puede incidir significativamente en la calidad del escrito, y repercutir positivamente en su calificación (Corcelles et al., 2013). Sin embargo, participar de la reflexión y del debate propio de la cultura científica no es tarea fácil. Lo agrava el hecho de que la enseñanza de la escritura académico-científica habitualmente no forma parte del currículo 
explícito de los estudios universitarios en contextos de habla hispana, y los profesores dan por supuesto que éstos ya disponen de los conocimientos necesarios para escribir cualquier texto (Lea \& Stierer, 2000; Carlino, 2003; Vargas Franco, 2005; López-Cózar et al., 2013; Castelló, 2015). Ello sucede a pesar de que la investigación ha puesto reiteradamente de manifiesto que los estudiantes, cuando se les pregunta, casi siempre expresan sus dificultades y solicitan ayuda para aprender a escribir (Castelló, Mateos, Castells, Iñesta, Cuevas \& Solé, 2012; Corcelles, Oliva, Castelló \& Milian, 2015). Así pues, la escritura de un artículo de investigación puede resultar muy difícil sin la ayuda de una intervención específicamente diseñada para este propósito.

En los últimos años, han sido varias las propuestas para mejorar la alfabetización académico-científica de los estudiantes universitarios (Moyano, 2004; Vargas Franco, 2005; Carlino, 2005; Dysthe, 2012; Corcelles et al., 2013). La mayoría de estos estudios se han centrado en los procesos y percepciones de los estudiantes de postgrado máster y doctorado- (Caffarella \& Barnett, 2000; Inman \& Silverstein, 2003; Aitchison \& Lee, 2006; Dysthe, Samara \& Westrheim, 2006; Castelló, Iñesta \& Monereo, 2009; Castelló, Iñesta \& Corcelles, 2013; entre otros) y son escasos los que han analizado su desarrollo en los estudios de grado (Todd et al., 2004; Greenbank \& Penketh, 2009; Heinze \& Heinze, 2009), especialmente en el ámbito español donde la implantación generalizada de la TFG es todavía reciente (Castelló et al., 2012; Corcelles et al., 2013).

Entre estos últimos, la mayoría se han ocupado del análisis de los planes de estudio y guías docentes, así como de la organización y evaluación de la materia (Vilardell, 2010; Rekalde, 2011; Bonilla \& López, 2012; Bonilla, Moraleda, Guerrero \& Guerrero, 2012; López et al., 2013; Sánchez Fernández, 2013; Martí, Marzal \& Medina, 2013). No obstante, son muy escasos los estudios que abordan la enseñanza de un género tan específico como el artículo de investigación (Castelló et al., 2013), y menos aun los que lo han hecho con estudiantes de grado en los estudios de educación.

\section{Método}

\subsection{Objetivos}

En este estudio presentamos una propuesta de enseñanza de la escritura académico-científica y analizamos empíricamente las percepciones de los estudiantes acerca de su desarrollo. Concretamente, los objetivos específicos del estudio empírico fueron:

a) Analizar las percepciones y los sentimientos de los estudiantes de grado acerca de la escritura de un artículo de investigación.

b) Describir las dificultades que experimentan durante este proceso de escritura académico-científica. 
c) Analizar sus percepciones respecto al aprendizaje realizado y las ayudas recibidas durante la intervención educativa en el contexto de la TFG.

\subsection{Diseño}

A partir de la implementación de la intervención, que presentamos en el apartado de resultados, se llevó a cabo un estudio cualitativo, basado en un diseño interpretativo (Bogdan \& Biklen, 2003), con la finalidad de describir e interpretar las respuestas abiertas de los estudiantes a una encuesta realizada durante y después del proceso de escritura para indagar sobre sus percepciones en relación al proceso de escritura académico-científica y a la misma intervención educativa.

\subsection{Participantes}

La muestra se compuso de 48 estudiantes agrupados en cuatro seminarios de $4^{\circ}$ curso, tres de educación infantil y uno de educación primaria (12 estudiantes por seminario; 71,4\% mujeres y 28,6\% hombres). La selección de los seminarios se realizó de manera intencional ya que los profesores de los mismos habían participado en una formación previa de enseñanza de la escritura académico-científica y consecuentemente seguían el modelo de intervención propuesto.

\subsection{Procedimiento}

La información se recogió a través de la administración de una encuesta on-line de respuestas abiertas, que los estudiantes respondieron en dos momentos: durante y al final del proceso de escritura del artículo de investigación (ver Anexo). Los estudiantes podían responder sin límite de tiempo ni de extensión.

Las cuestiones incluidas en la encuesta inicial hacían referencia a las 'percepciones y sentimientos sobre la escritura del artículo de investigación’ en el contexto de la TFG (objetivo a). En la encuesta final, se incluyeron cuestiones relativas a su valoración del proceso, concretamente en relación a las 'dificultades experimentadas' (objetivo b) y el 'aprendizaje realizado' así como las 'ayudas recibidas' (objetivo c).

Para el análisis de los datos, se procedió de la siguiente manera. En primer lugar se delimitó la unidad de análisis, los 'enunciados', entendidos como unidades mínimas de significado sobre un mismo tema. La adopción de esta unidad de análisis implica que cada una de las respuestas de los participantes podía contener más de un enunciado. En total se obtuvieron un total de 526 enunciados. En segundo lugar, los enunciados se clasificaron en dimensiones según su relación con los objetivos del estudio; así se distinguieron aquellos enunciados que hacían referencia a las 'percepciones y sentimientos de los estudiantes sobre la escritura de un artículo de investigación', los que trataban sobre las 'dificultades experimentadas durante la escritura' y aquellos relativos al 'aprendizaje realizado y las ayudas recibidas' durante el proceso de escritura. 
En tercer lugar, se procedió al establecimiento de categorías temáticas. Para ello, todos los autores leímos de nuevo de forma iterativa la totalidad de los enunciados incluidos en cada dimensión y discutimos las relaciones temáticas entre los mismos hasta consensuar un sistema de categorías que representara adecuadamente su diversidad y variabilidad. Una vez establecido el sistema de categorías, tres investigadores conocedores de los objetivos y el marco teórico de la investigación, analizaron un tercio de los enunciados de forma independiente. Su grado de acuerdo fue del $92 \%$. Los pocos casos en los que se observaron discrepancias $(8 \%$ de los enunciados) se discutieron hasta conseguir un acuerdo. Una vez garantizada la fiabilidad de las categorías, el resto de enunciados fue analizado de manera equitativa entre los investigadores.

\section{Resultados}

Teniendo en cuenta los objetivos del estudio, presentamos en primer lugar las características de la intervención realizada, y en segundo lugar, los resultados respecto a las percepciones y sentimientos de los estudiantes acerca de la escritura del artículo de investigación (objetivo a); las dificultades en la escritura de un artículo de investigación (objetivo b), y finalmente las percepciones relativas al de aprendizaje y a las ayudas recibidas en la intervención educativa (objetivo c).

\subsection{Descripción de la intervención educativa y de la secuencia de enseñanza de la escritura académico-científica}

La intervención se realizó durante un curso académico en el espacio denominado 'seminario de TFG' de cuarto curso de los estudios de grado de educación infantil y grado de educación primaria de la Universidad Ramon Llull. Participaron cuatro seminarios, tres de educación infantil y uno de educación primaria. Cada seminario estaba formado por grupos de 12 a 15 estudiantes que se reunían con un profesor una vez a la semana en sesiones de $2 \mathrm{~h}$ (que se complementaban con atenciones individuales) a lo largo de todo un curso académico (durante los dos semestres), con un total de 16 sesiones. En todos los seminarios se siguió una misma estructura de actividades para guiar la escritura del artículo de investigación. En el primer semestre, las ayudas se dirigieron a la elección del tema a investigar, la selección de lecturas, la escritura del marco teórico y del diseño de la investigación. En el segundo semestre, los estudiantes llevaron a cabo la recogida y el análisis de datos junto con la escritura de los resultados y la discusión del artículo. Durante el proceso de escritura se utilizó la revisión colaborativa de los textos por parejas de tal manera que cada estudiante fue autor de su texto y revisor del texto de su compañero. La posibilidad de desarrollar esta doble mirada, desde la posición de autor y de revisor, ha sido destacada como eficaz a la hora de mejorar la calidad del texto escrito y superar problemas recurrentes 
durante el proceso de composición como los bloqueos o la escasez de revisiones (DiPardo \& Freedman, 1988; Castelló et al., 2012; Corcelles et al., 2013).

Todos los estudiantes inscritos en los seminarios de la TFG debían elaborar un texto que cumpliese los estándares de los textos académico-científicos de su disciplina de referencia, se ajustase a la estructura habitual de los artículos de investigación (título, resumen, palabras clave, introducción, método, resultados, conclusiones y referencias bibliográficas) (Swales, 2004) y al formato de la normativa APA22.

Para paliar los problemas asociados a la escritura de un artículo de investigación destacados por la literatura, la intervención educativa partió de tres principios:

a) Ampliar el conocimiento de los estudiantes sobre los textos científicos, en especial los artículos de investigación.

b) Facilitar el conocimiento y la conciencia de los estudiantes sobre el propio proceso de composición y dotarles de recursos para gestionarlo y regularlo.

c) Promover la intertextualidad y la interactividad a lo largo del proceso de composición mediante la revisión colaborativa.

En último término, a través de la intervención educativa se pretendía que los estudiantes comprendieran el sentido que tienen los textos científicos en la comunidad educativa, así como las características de este tipo de género. También se propició que aprendieran a dialogar y relacionarse con otros autores haciendo que emergiera su 'voz' en el texto (Castelló et al., 2011).

A partir del modelo de enseñanza de la escritura desarrollado por Castelló (2002) dividimos la secuencia de actividades vinculadas a la enseñanza del género del artículo de investigación en tres momentos del proceso de enseñanza-aprendizaje:

\section{I) Antes de la escritura}

A lo largo del primer semestre, los estudiantes desarrollaron actividades que implicaban 'leer para escribir' y 'hablar sobre la escritura'.

La primera de estas actividades - 'leer para escribir'-, supuso leer artículos científicos similares a los que los estudiantes debían escribir con el objetivo, no sólo de comprender la información, sino sobre todo de analizar cómo estaban escritos. Se buscaron y discutieron ejemplos de cómo los autores habían estructurado el artículo, utilizado los recursos discursivos o las citas. Para facilitar este análisis, se proporcionó a los alumnos una guía a partir de las siguientes dimensiones:

- Organización de la información: cómo empieza el texto, cómo acaba, qué conectores se utilizan; cómo se produce la progresión de las ideas en torno a 
uno o varios ejes estructuradores, cuándo y cómo se establecen los objetivos, cuál es el nicho de la investigación y cuándo y cómo se comunica al lector.

- Posicionamiento del autor: recursos para enfatizar y/o matizar la información ${ }^{3}$, marcadores de actitud, auto-referencias y recursos para implicar al lector.

- Intertextualidad: tipo de citas utilizadas (directas o indirectas) y función de las mismas (identificación, diferenciación y evaluación).

Para complementar la información, se proporcionó un dossier con explicaciones y ejemplos específicos de las características propias de este género ${ }^{4}$.

El segundo grupo de actividades, a las que de forma genérica denominamos 'hablar para escribir', se orientaron tanto a facilitar la tarea de escritura como a hacer más explícito y visible el proceso de composición a seguir. Mediante la 'escritura elaborativa', se discutió con el profesor y los compañeros cómo se integran diversas fuentes de información sin que el texto pierda su estructura o cómo se conectan y se relacionan de forma coherente estas fuentes con los propios objetivos.

Por último, y ya en el capítulo de actividades que implican 'escribir sobre la propia escritura', los estudiantes realizaron un diario sobre el proceso seguido, los problemas enfrentados, los sentimientos experimentados y las soluciones empleadas. Como hemos argumentado en otros trabajos, revisar estos diarios al cabo de una o varias semanas de redacción es sumamente ilustrativo y facilita la toma de conciencia acerca del propio proceso de composición (Castelló, 2002).

\section{II) Durante la escritura}

A mediados del primer semestre los estudiantes empezaron a escribir sus textos con la ayuda de la guía de escritura y los diarios de escritura. El profesor explicó las características de la revisión colaborativa, constituyó las parejas de revisión a partir de criterios de afinidad temática y rendimiento similar y se formó a los estudiantes acerca de lo que se esperaba de ellos como tutores y como escritores y de la tipología de sugerencias que podían proporcionar a sus compañeros.

Se planificaron tres entregas del texto escrito aproximadamente cada mes y medio: una inicial, en la que se esperaba que los estudiantes tuvieran un borrador, como mínimo, del apartado de la introducción o fundamentación teórica del artículo y de la descripción del método, una intermedia con el artículo casi completo, o al menos, con la introducción, método y resultados, y una final con el artículo ya terminado en su versión final (conclusiones y discusión). Después de cada entrega y antes de la sesión de seminario, los estudiantes, organizados en parejas, tenían que revisar on-line el texto de su compañero. Las parejas se ofrecían de manera recíproca sugerencias de cambio respecto al texto escrito a partir de comentarios que inserían en el texto usando los 
comentarios del procesador de textos del Word y que discutían posteriormente en las sesiones presenciales de seminario. Por otra parte, el profesor también realizaba sus comentarios sobre el texto ya revisado por el compañero que además podían complementarse con tutorías individuales y ofrecía al estudiante una evaluación individual mediante una rúbrica basada en unos criterios negociados previamente con los estudiantes (Corcelles et al., 2013).

\section{III) Después de la escritura}

Una vez recibidas las sugerencias del compañero y del profesor, los estudiantes revisaban sus textos y los modificaban según su criterio; estas revisiones se incorporaban a la siguiente entrega del texto

\subsection{Percepciones y sentimientos asociados a la escritura del artículo de investigación}

Los resultados del análisis de la encuesta administrada durante y al final del proceso de escritura mostraron que solo un $3,3 \%$ de los enunciados de los estudiantes aludieron a una percepción negativa del proceso de escritura en el contexto de la TFG. Entre el resto de los enunciados (96,7\%), identificamos tres categorías que ilustran formas cualitativamente diferentes de entender y dar sentido a la escritura del artículo de investigación (ver Tabla1):

Tabla 1. Percepciones de los estudiantes sobre la escritura del artículo de investigación.

\begin{tabular}{|c|l|c|c|c|}
\hline \multicolumn{1}{|c|}{ Percepciones sobre la escritura } & Durante & Final & Total \\
\hline \multirow{7}{*}{ Positivas } & $\begin{array}{l}\text { Profesional y académica: la } \\
\text { escritura como herramienta para } \\
\text { aprender a investigar y desarrollar } \\
\text { el rol de docente investigador. }\end{array}$ & $16(17.8 \%)$ & $37(41,1 \%)$ & $53(58,9 \%)$ \\
\cline { 2 - 5 } & $\begin{array}{l}\text { Crecimiento personal: la escritura } \\
\text { como herramienta para } \\
\text { profundizar en un tema de interés. }\end{array}$ & $6(6,7 \%)$ & $18(20 \%)$ & $24(26,7 \%)$ \\
\cline { 2 - 5 } & $\begin{array}{l}\text { Curricular: la escritura como } \\
\text { herramienta para aplicar o } \\
\text { demostrar los conocimientos } \\
\text { adquiridos. }\end{array}$ & $9(10 \%)$ & $1(1,1 \%)$ & $10(11,1 \%)$ \\
\cline { 2 - 5 } & & $31(34,5 \%)$ & $56(62,2 \%)$ & $87(96,7 \%)$ \\
\hline Negativas & $2(2,2 \%)$ & $1(1,1 \%)$ & $3(3,3 \%)$ \\
\hline \multicolumn{2}{|l|}{} & & Total & $(100 \%)$ \\
\hline
\end{tabular}

a. Profesional y académica: Bajo esta denominación, más frecuente al finalizar el proceso, se incluyeron aquellos estudiantes que percibieron la escritura del artículo de investigación como una herramienta para aprender a investigar y a desarrollar su rol de docentes-investigadores, tal como ilustran los ejemplos siguientes: "[...] como maestras tenemos que practicar la autorreflexión y compartirla con la comunidad educativa para innovar y avanzar. La TFG sirve para realizar una investigación-acción, compartir los conocimientos y 
reflexionar sobre la práctica" (S1P1a); o "Aprender a escribir artículos científicos es una buena idea para que en un futuro próximo podamos participar en revistas educativas" (S2P2a).

b. Crecimiento personal: En este caso, la categoría alude a la escritura como una herramienta para profundizar en un tema de interés personal que, consecuentemente, impacta en el propio desarrollo profesional. El ejemplo siguiente resulta ilustrativo: "El sentido de la TFG es convertirse en experto sobre un tema y profundizar en estudios previos" (S2P6a).

c. Curricular: Esta categoría, especialmente frecuente durante la escritura del artículo de investigación, implica que la actividad de escribirse considera parte del currículo y consiste en la aplicación y demostración de los conocimientos adquiridos, tal como se expresa el siguiente comentario: "Utilizar los contenidos trabajados durante los cuatro años de carrera así como también dejar constancia de la capacidad de reflexión que tiene cada uno de nosotros" (S2P1a).

Tabla 2. Sentimientos asociados a la escritura del artículo de investigación.

\begin{tabular}{|l|r|r|r|}
\hline \multicolumn{1}{|c|}{ Sentimientos } & \multicolumn{1}{c|}{ Durante } & \multicolumn{1}{c|}{ Final } & \multicolumn{1}{c|}{ Total } \\
\hline Satisfacción, superación y/o motivación & $8(11,4 \%)$ & $30(42,9 \%)$ & $38(54,3 \%)$ \\
\hline Ansiedad & $12(17,1 \%)$ & $11(15,7 \%)$ & $23(32,9 \%)$ \\
\hline Desmotivación y/o desorientación & $7(10 \%)$ & $2(2,9 \%)$ & $9(12,9 \%)$ \\
\hline Total & $27(38,6 \%)$ & $43(61,4 \%)$ & $70(100 \%)$ \\
\hline
\end{tabular}

En referencia a los sentimientos (ver Tabla 2), en general la escritura del artículo de investigación se asoció a sentimientos positivos de satisfacción, superación y motivación (54,3\%), especialmente al finalizar el proceso, pero también a sentimientos negativos de ansiedad (32,9\%), y en menor medida de desmotivación y/o desorientación $(12,9 \%)$ sobre todo durante el proceso. Los siguientes enunciados ilustran estos sentimientos:

"Es muy interesante hacer un texto de esta magnitud, te obliga a hacer un esfuerzo importante pero muy provechoso. A lo largo del proceso ha habido momentos de total claridad y momentos de mucha confusión, pero el resultado final ha sido muy satisfactorio" (S3P5b).

"La sensación es como si estuviera surfeando: unos momentos estoy arriba de la ola, y no paro de trabajar y solucionar los problemas, y después estoy abajo y me siento desanimada, inquieta (sentimiento que tengo constantemente), muy nerviosa, y me cuesta superar los retos" (S3P2b). 
En ocasiones, los estudiantes experimentaron tanto sentimientos positivos como negativos a lo largo de la escritura del artículo de investigación, como evidencia el siguiente ejemplo:

"Durante todo el proceso he pasado por diferentes etapas. Ha habido momentos, sobre todo al principio, en los que me he sentido un poco perdida e insegura. Pero a medida que iba avanzando fui adquiriendo más seguridad y más conciencia de que sí lo podría hacer bien. Creo que ha sido interesante realizar este proyecto, ya que en un principio lo concebía como imposible y me he dado cuenta de que poco a poco iba adquiriendo forma y mis sentimientos iban cambiando" (S3P1a).

\subsection{Dificultades en la escritura del artículo de investigación}

Los estudiantes percibieron la escritura del artículo de investigación como una tarea compleja como lo ilustran el $97,5 \%$ de los enunciados (ver Tabla 3), especialmente al finalizar su proceso de escritura (60,4\%).

Tabla 3. Frecuencia de dificultades percibidas por los estudiantes ante la escritura del artículo de investigación.

\begin{tabular}{|c|l|c|c|c|}
\hline $\begin{array}{c}\text { Dificultades } \\
\text { TFG }\end{array}$ & $\begin{array}{c}\text { Durante el } \\
\text { proceso }\end{array}$ & $\begin{array}{c}\text { Final del } \\
\text { proceso }\end{array}$ & Total \\
\hline \multirow{3}{*}{ Sí } & $\begin{array}{l}\text { Género y escritura } \\
\text { académica }\end{array}$ & $33(20,8 \%)$ & $42(26,5 \%)$ & $75(47,3 \%)$ \\
\hline \multirow{5}{*}{} & $\begin{array}{l}\text { Proceso de } \\
\text { investigación }\end{array}$ & $16(10,1 \%)$ & $36(22,6 \%)$ & $52(32,7 \%)$ \\
\cline { 2 - 5 } & Tiempo & $9(5,6 \%)$ & $14(8,8 \%)$ & $23(14,4 \%)$ \\
\cline { 2 - 5 } & Otros & $1(0,6 \%)$ & $4(2,5 \%)$ & $5(3,1 \%)$ \\
\cline { 2 - 5 } & Total Sí & $59(37,1 \%)$ & $96(60,4 \%)$ & $155(97,5 \%)$ \\
\hline No & Total No & $1(0,6 \%)$ & $3(1,9 \%)$ & $4(2,5 \%)$ \\
\hline Total & & $60(37,7 \%)$ & $99(62,3 \%)$ & $159(100 \%)$ \\
\hline
\end{tabular}

Un análisis detallado de estos enunciados nos permitió agrupar las dificultades en tres categorías (de mayor a menor frecuencia):

- Dificultades relacionadas con las características del género artículo de investigación y su escritura: los estudiantes mencionaron, tanto durante como al finalizar el proceso de escritura, su falta de experiencia y conocimiento del género y las convenciones y exigencias propias de la escritura científica, como ilustra la cita siguiente: "No me ha resultado nada fácil escribir este tipo de texto; nunca había trabajado en un artículo de investigación pues los temas educativos suelen ser más divulgativos" (S1P4a). También señalaron dificultades en la redacción, síntesis e integración de la información: "He tenido dificultades sobretodo relacionadas con la redacción del artículo: seguir la estructura, no sintetizar suficiente o sintetizar en exceso, utilizar los tiempos verbales inadecuados o expresiones demasiado coloquiales" (S4P1b). 
- Dificultades relacionadas con el proceso de investigación: en este caso los enunciados hacían referencia a la identificación del tema, la búsqueda y selección de la información, la recogida y análisis de los datos, como en el caso siguiente: "Desde el principio me costó mucho escoger un tema, buscar y seleccionar la información, enfocar el trabajo [...] son retos que requieren esfuerzo y dedicación” (S1P6a). En una línea similar, otra estudiante comentó: "He encontrado dificultades para encontrar profesionales que quisieran participar en el estudio y también para analizar los datos de las entrevistas, para relacionar todos los datos para sacar unas conclusiones" (S2P4b).

- Dificultades relacionadas con la gestión del tiempo: en este caso, muchos de los enunciados aludieron a sensaciones de estrés, como en el caso siguiente: "Difícil, duro y cansado. Me ha provocado angustia porque tenemos mucho trabajo y poco tiempo" (S1P6a). En ocasiones, la dificultad en la gestión del tiempo se atribuyó además a causas curriculares: "La falta de tiempo por los trabajos de otras asignaturas. También cabe tener en cuenta que en cuarto curso es cuando tenemos más prácticas" (S2P1b).

\subsection{Percepciones relativas al aprendizaje $y$ a las ayudas recibidas en la intervención educativa}

En referencia a los aprendizajes, los estudiantes (ver Tabla 4) destacaron que la TFG les ayudó principalmente a mejorar sus habilidades de escritura académicocientífica (un 46,4\% de los enunciados). El ejemplo siguiente es ilustrativo al respecto: "He aprendido a citar y referenciar en formato APA, la estructura de los artículos de investigación y qué debe contener cada apartado y de qué manera hay que organizar la información para que el texto sea coherente y siga un hilo conductor claro" (S4P2a).

Tabla 4. Percepción de los estudiantes sobre los aprendizajes propiciados a lo largo de la intervención educativa.

\begin{tabular}{|c|c|}
\hline Aprendizajes durante la intervención educativa & Final \\
\hline Escritura académico-científica & $32(46,4 \%)$ \\
\hline Proceso de investigación & $16(23,2 \%)$ \\
\hline Crecimiento personal & $11(15,9 \%)$ \\
\hline Aprendizaje en relación al dominio de un tema & $10(14,5 \%)$ \\
\hline Total & $69(100 \%)$ \\
\hline
\end{tabular}

En segundo lugar, manifestaron que fundamentalmente aprendieron a desarrollar todas las actividades que conlleva un proceso de investigación $(23,2 \%)$, como ilustra el comentario siguiente: "He aprendido cómo buscar información contrastada, crítica y científica y, por lo tanto cómo formar parte de un grupo de investigación o escribir cualquier documento de carácter científico" (S3P2b), y a desarrollar una nueva identidad relacionada con el rol de investigador: 
"He descubierto que me gusta llevar a cabo experiencias empíricas que puedan dar respuesta a hipótesis planteadas, me he encontrado a mí misma realizando todo un trabajo de investigación, lo que ha despertado en mí las ganas para continuar investigando" (S2P7a).

Para muchos estudiantes la realización de la investigación y la escritura del artículo de investigación durante la TFG conllevó también un crecimiento personal (15,9\%) que vincularon al desarrollo de actitudes y habilidades como la constancia, la perseverancia y el esfuerzo de superación, como ilustra el siguiente ejemplo: "He aprendido a ser perseverante, con esfuerzo y constancia. Luchar para seguir hacia delante y no rendirme, pues en muchas ocasiones he estado al límite” (S1P6b).

Por último, los estudiantes manifestaron que también aprendieron sobre contenidos específicos (14,5\%), lo que vincula la escritura académico-científica con el aprendizaje disciplinar, como demuestra el ejemplo siguiente: "He aprendido mucho de la temática estudiada, de muchos autores que han tenido preocupaciones similares" (S3P5b).

En referencia a la valoración de las ayudas que recibieron durante la intervención educativa, la gran mayoría de los enunciados (90,7\%) aludían a una valoración positiva de las ayudas proporcionadas durante la intervención. Los estudiantes destacaron el hecho de sentirse acompañados y orientados durante todo el proceso de escritura como ilustra el comentario siguiente: "Mi valoración es muy positiva, me he sentido apoyada y acompañada en todo momento, se nos ha orientado y ofrecido mucha ayuda" (S2P2b).

Los escasos enunciados que referían una valoración media o baja se relacionaron con aspectos de tiempo o con el incumplimiento de sus expectativas, como muestra el siguiente ejemplo: "La valoración es buena aunque en algunos momentos esperaba más y por la falta de tiempo quedaban dudas en el aire” (S1P5a).

Tabla 5. Valoración de la calidad de la retroalimentación del profesor y del compañero/a.

\begin{tabular}{|l|l|l|l|}
\hline \multicolumn{2}{|c|}{ Profesor } & \multicolumn{2}{c|}{ Compañero/a } \\
\hline Positiva & $39(100 \%)$ & Positiva & $38(90,5 \%)$ \\
\hline Revisar errores y avanzar & $34(87,2 \%)$ & Revisar errores y avanzar & $31(73,9 \%)$ \\
\hline Soporte de un experto & $5(12,8 \%)$ & Soporte emocional & $4(9,5 \%)$ \\
\hline & & Audiencia & $3(7,1 \%)$ \\
\hline Negativa & 0 & Negativa & $4(9,5 \%)$ \\
\hline & & No experto & $4(9,5 \%)$ \\
\hline
\end{tabular}

Respecto a la calidad de la retroalimentación del profesor y de los compañeros facilitaron mediante la revisión colaborativa (ver Tabla 5) los resultados muestran que se valoró como positivo en ambos casos (100\% y 90,5\% respectivamente). El ejemplo siguiente es representativo de estas valoraciones: "Valoro muy positivamente el 
feedback de la profesora y del compañero porque me ayudaba a reflexionar y a detectar errores o a identificar formas mejores de redactar que las que yo empleaba" (S4P1b)

La retroalimentación del profesor fue destacada por su impacto en la revisión de los errores y el avance del proyecto $(87,2 \%)$ y, en menor medida, por su condición de experto $(12,8 \%)$. En el caso de la revisión colaborativa, la retroalimentación de los compañeros se valoró también su influencia en la revisión de los errores y el avance del proyecto $(73,9 \%)$ y en menor medida, por la provisión de soporte emocional $(9,5 \%)$ y de una audiencia real $(7,1 \%)$.

Los enunciados siguientes constituyen ejemplos representativos de los comentarios relativos al soporte emocional: "El feedback de la compañera me ha ido muy bien sobre todo a nivel emocional. El de la profesora me ha sido útil sobre todo para ordenar las ideas y para saber hacia dónde avanzar" (S1PGa); o de la consideración del compañero como un lector potencial: "Me ha resultado muy útil el feedback de mi compañero ya que él se leía mi artículo como una persona externa y sus comentarios servían para detectar ideas confusas que pensaba que se entendían bien pero que quizás no estaban suficientemente claras" (S2P3b).

Tabla 6. Valoración del rol de revisor/ a en la revisión colaborativa.

\begin{tabular}{|l|l|}
\hline Positivo: Aprendizaje & $45(78,9 \%)$ \\
\hline Aprender a escribir & $21(36,8 \%)$ \\
\hline Aprender a revisar y dar soporte al compañero & $16(28,1 \%)$ \\
\hline Aprender a trabajar en equipo & $4(7 \%)$ \\
\hline Adquirir nuevos conocimientos sobre un tema & $4(7 \%)$ \\
\hline Dificultades: inseguridad en ofrecer retroalimentación & $12(21,1 \%)$ \\
\hline
\end{tabular}

Finalmente, respecto a las percepciones de los estudiantes sobre la revisión colaborativa (ver Tabla 6), los resultados indican también una valoración positiva del rol de revisores, ya que la mayoría de los enunciados $(78,9 \%)$ indicaron que la revisión del texto del compañero fue útil para escribir mejor $(36,8 \%)$ pero también para revisar y dar apoyo emocional a un compañero $(28,1 \%)$. Los siguientes ejemplos ilustran estos aspectos: "Desempeñando el rol de revisor he aprendido a valorar los textos desde otra perspectiva y otros estilos de escritura y a la vez me ha aportado nuevas ideas para mi texto" (S3P2a). "Actuando como revisor he aprendido a tener más tacto para decir las cosas que se podrían o deberían cambiar, incitar a hacer cambios sin decirlo directa o impositivamente y a apoyar al escritor" (S1p1a).

Por otro lado, aunque en menor medida, los estudiantes valoraron que la revisión colaborativa promovió el trabajo en equipo (7\%), como ilustra la cita siguiente: "Mediante el desempeño del rol de revisor he aprendido a involucrarme con mi compañero y a propiciar un trabajo colaborativo" (S2P6b); o la oportunidad de conocer un tema distinto al suyo $(7 \%)$ : 
"He aprendido aspectos sobre su tema, aunque al principio, al no ser un tema familiar para mí, me costaba ubicarme y valorar el texto. Pero finalmente al leerlo tantas veces de manera detenida para identificar errores te vas haciendo más conocedor del tema y esto facilita la tarea" (S3P6a).

Sin embargo, cabe tener en cuenta también que algunos estudiantes manifestaron dificultades en la revisión colaborativa debido a la inseguridad que sentían al no ser expertos en el tema elegido por el compañero/a $(21,1 \%)$, como vemos en los siguientes ejemplos: "En algunos momentos me ha resultado complicado debido a mi falta de experiencia y al poco dominio de la temática de mi compañero" (S2P6b); "Al inicio me resultó muy difícil ya que no estoy acostumbrada a revisar a otro compañero y me resultaba difícil identificar sus errores en la escritura pues soy consciente que yo tampoco soy una buena escritora" (S3P3a).

\section{CONCLUSIONES}

En este estudio hemos descrito una propuesta de enseñanza de la escritura académico-científica, concretamente del género artículo de investigación, basada en la revisión colaborativa como estrategia didáctica y hemos indagado empíricamente sobre las percepciones de los estudiantes respecto a dicha intervención y su proceso de escritura.

Los resultados indican, en primer lugar, que la mayoría de los estudiantes percibió positivamente la realización de la TFG, algo que ha sido también corroborado por algunos estudios previos como avanzábamos en la introducción (Ryder, 2004; Seymour et al., 2004; Corcelles et al., 2013). La mayoría de los estudiantes percibió la escritura del artículo de investigación como una herramienta para participar en su comunidad profesional y desarrollar un rol de docente-investigador, superando las tensiones que supuso la adopción de este nuevo rol en relación al rol habitual de estudiante universitario, y evidenciando así la potencial contribución de la escritura de un artículo de investigación durante la TFG en el desarrollo de una nueva faceta de su identidad docente relacionada con la investigación (Giroux, 1990; Zeichner, 1993; Schön, 2002; Latorre, 2003; Zeichner \& Liston, 2014).

En segundo lugar, los resultados muestran que la gran mayoría de los estudiantes coincidieron en considerar la escritura académico-científica como una actividad compleja que comportó un nivel elevado de dificultad. Concretamente, la principal dificultad se relacionó con el desconocimiento del género del artículo científico y la falta de habilidades de escritura, lo que pone de manifiesto la necesidad de incrementar la formación previa de los estudiantes universitarios en este aspecto garantizando la enseñanza de este tipo de escritura a lo largo de toda la formación universitaria y no exclusivamente durante el primer o último curso del grado, de tal manera que los estudiantes dispongan de herramientas imprescindibles para 
desarrollar su competencia investigadora (Lea \& Stierer, 2000; Bazerman et al., 2005; Carlino, 2005; Corcelles et al., 2015). Otras dificultades percibidas por los estudiantes se vincularon al proceso propio de investigación (selección del tema, búsqueda de la información, recogida y análisis de los datos), así como a la gestión del tiempo, aspectos también reportados en estudios anteriores (Todd, et al., 2004; Harrison \& Whalley, 2006, 2008; López-Cózar et al., 2013).

Por otro lado, confrontar estas dificultades, propias de un trabajo complejo como la escritura de un artículo de investigación, implicó la gestión de sentimientos negativos pero también positivos en distintos momentos del proceso. La mayoría de los estudiantes experimentaron, en alguna ocasión, emociones negativas como la ansiedad o sentimientos de desorientación y desmotivación. Sin embargo, al finalizar el proceso, a pesar de que en algunos casos la ansiedad persistía debido a la exigencia de las revisiones, la mayoría se manifestaron satisfechos por la superación del reto. Los escasos trabajos que han analizado los sentimientos asociados a la escritura de investigación también apuntan a esta mezcla de ansiedad y satisfacción que se explica por el carácter altamente demandante que supone la elaboración de textos científicos (Gallego, Castelló \& Badia, 2015).

Para la gran mayoría de los estudiantes la experiencia generó diversos aprendizajes. Así, reportaron mejoras en la escritura académico-científica que se concretaron en un uso más exigente y riguroso del lenguaje como requisito para participar en su comunidad profesional y construir conocimiento disciplinar. También manifestaron mejoras en su competencia como investigadores, como las relacionadas con actitudes y habilidades de crecimiento personal. Tal como algunos estudios recientes han puesto de manifiesto, estos aspectos influyen en el desarrollo de la identidad y especialmente con la construcción del rol del docente-investigador, aspecto que, como hemos mencionado, consideramos muy relevante para la formación del futuro docente (Beijaard et al., 2000; Aguayo et al., 2014).

En tercer lugar, respecto a la intervención realizada, la gran mayoría de estudiantes percibieron positivamente la calidad de las ayudas, tanto del profesor como de los compañeros, ofrecidas mediante la revisión colaborativa. El hecho de que la valoración de la revisión colaborativa tenga que ver con criterios de calidad y eficiencia (revisar los errores y avanzar) además de emocionales y contextuales (audiencia real), así como con una valoración positiva de su rol como revisores para aprender a escribir y mejorar sus propios textos indica que el sentido que los estudiantes otorgaron a la estrategia didáctica de la revisión colaborativa coincide con los resultados de estudios anteriores y avala su eficacia (DiPardo \& Freedman, 1988; Heylings \& Tariq, 2001; Inman \& Silverstein, 2003; Orsmond et al., 2004; Vargas Franco, 2005; Castelló et al., 2012; Corcelles et al., 2013). La inseguridad a la que aludieron algunos estudiantes al ejercer su rol de revisores ha sido también discutida en otros estudios en los que 
además se observó una cierta dificultad a la hora de ofrecer y encajar las críticas que no observamos en nuestro estudio (Aitchison \& Lee, 2006; Dysthe, 2012).

Así pues, podemos concluir que la intervención fue útil para ayudar a estos estudiantes a confrontar las dificultades, a regular sus emociones durante el proceso y a vincular el trabajo realizado y la escritura académico-científica con sentimientos positivos.

No queremos finalizar sin matizar los resultados a la luz de las limitaciones del estudio. Somos conscientes de que el hecho de partir de una encuesta realizada en dos momentos distintos de la intervención, con una muestra inferior a cincuenta estudiantes de educación de una misma universidad limita el alcance de los resultados que podrían ser muy diferentes si se modificaran las condiciones contextuales. Además, el hecho de que no hayamos analizado la calidad de las producciones textuales, podría también aducirse como una limitación del estudio. Sin embargo, dadas las razones de espacio disponible y la existencia de otros trabajos recientes en los que precisamente analizamos el cambio y la mejora de los textos (Castelló et al., 2012; Corcelles et al., 2013), nos pareció que era más pertinente centrarnos en las percepciones de los estudiantes de acuerdo a los objetivos aquí defendidos. Teniendo en cuenta la escasez de investigaciones en relación con las percepciones de los estudiantes sobre la escritura de textos académico-científicos en el grado y particularmente en la realización de la TFG, consideramos que este tipo de trabajos puede contribuir a comprender mejor la complejidad y exigencia que conlleva la realización de un artículo de investigación en el contexto de grado y, de esta forma, incidir en la mejora de su enseñanza. Además, esperamos que la propuesta de intervención que hemos presentado y analizado sirva de acicate no sólo para el desarrollo de propuestas similares sino, sobre todo, para enriquecer el debate acerca de cómo podemos formar a los futuros docentes en la escritura de géneros de investigación entendiendo que se trata de herramientas imprescindible para el desarrollo de su competencia investigadora, de su identidad como docentesinvestigadores y, en definitiva, para la mejora de la calidad educativa (Zeichner, 1993; Latorre, 2003; Zeichner \& Liston, 2014).

\section{REFERENCIAS BIBLIOGRÁFICAS}

Aitchison, C. \& Lee, S. (2006). Research writing: Problems and pedagogies. Teaching in Higher Education, 11(3), 265-278.

Aguayo, M., Castelló, M. \& Monereo, C. (2014). La identidad del académico de enfermería: Entre la docencia y la investigación. Texto \& Contexto Enfermagem, 23(2), 241- 249. 
Bazerman, C., Little, J., Chavkin, T., Fouquette, D., Bethel, L. \& Garufis, J. (2005). Reference guide to writing across the Curriculum. Reference guides to rhetoric and composition. West Lafayette, Indiana: Parlor Press and WAC Clearinghouse [en línea]. Disponible en: http://wac.colostate.edu/books/bazerman_wac/

Beijaard D., Verloop N. \& Vermunt J. D. (2000). Teachers' perceptions of professional identity: An exploratory study from a personal knowledge perspective. Teaching and Teacher Education, 16(7), 749-64.

Bogdan. R. \& Biklen, S. (2003). Qualitative research for education: an introduction to theories and methods. Nueva York: Allyn \& Bacon.

Bonilla, M. I. \& López, C. M. (2012). Evaluación de competencias en el Trabajo Fin de Grado en Administración y Dirección de Empresas: Una propuesta de la Facultad de Ciencias Sociales de Talavera de la Reina. UCLM. Revista de Formación e Innovación educativa Universitaria, 5(4), 241-253.

Bonilla, M. J., Moraleda, L. F., Guerrero, C. V. \& Guerrero, T. V. (2012). Análisis del proceso de evaluación del Trabajo Fin de Grado en las nuevas titulaciones. Educade: Revista de educación en contabilidad, finanzas y administración de empresas, 3, $5-21$.

Caffarella, R. \& Barnett, B. (2000). Teaching doctoral students to become scholarly writers: The importance of giving and receiving critiques. Studies in Higher Education, 25(1), 39-52.

Carlino, P. (2003). Alfabetización académica: Un cambio necesario, algunas alternativas posibles. Educere Investigación, 6(20), 409-420.

Carlino, P. (2005). Escribir, leer y aprender en la universidad. Una introducción a la alfabetización académica. Buenos Aires: Fondo de Cultura Económica.

Castelló, M. (2002). De la investigación sobre el proceso de composición a la enseñanza de la escritura. Revista Signos. Estudios de Lingüística, 35(51-52), 149162.

Castelló, M. (Coord.) (2015). La investigación sobre escritura académica en los procesos de enseñanza-aprendizaje en la universidad española. Cultura $y$ Educación: Revista de teoría, investigación y práctica, 27(3), 471-476.

Castelló, M. \& Iñesta, A. (2012). Texts as artifacts-in-activity: Developing authorial identity and academic voice in writing academic research papers. En M. Castelló \& C. Donahue (Eds), University writing: Selves and texts in academic societies (pp. 177-200). Londres: Emerald Group. 
Castelló, M., Iñesta, A. \& Monereo, C. (2009). Towards self-regulated academic writing: An exploratory study with graduate students in a situated learning environment. Electronic Journal of Research in Educational Psychology, 7(3), 1107 1130.

Castelló, M., Iñesta, A. \& Corcelles, M. (2013). Ph. D. students' transitions between academic and scientific writing identity: Learning to write a research article. Research in the Teaching of Writing, 47(4), 442-477.

Castelló, M., Corcelles, M., Iñesta, A., Vega, N. \& Bañales, G. (2011). La voz del autor en la escritura académica: Una propuesta para su análisis. Revista Signos. Estudios de Lingüistica, 44(76), 105-117.

Castelló, M., Iñesta, A., Pardo, M., Liesa, E. \& Martínez-Fernández, R. (2012). Tutoring the end-of-studies dissertation: Helping psychology students find their academic voice. Higher Education, 63(1), 97-115.

Castelló, M., Mateos, M., Castells, N., Iñesta, A., Cuevas, I. \& Solé, I. (2012). Academic Writing Practices in Spanish Universities. Electronic Journal of Research in Educational Psychology, 10(2), 569-590.

Corcelles, M., Cano, M., Bañales, G. B. \& Vega, N. (2013). Enseñar a escribir textos científico-académicos mediante la revisión colaborativa: El trabajo final de grado en Psicología. REDU: Revista de Docencia Universitaria, 11(1), 79-104.

Corcelles, M., Oliva, A., Castelló, M. \& Milian, M. (2015) Escribir en la Universidad: ¿Nos entendemos? Cultura y Educación: Revista de teoría, investigación y práctica, 27(3), 550-566.

Derounian, J. (2011). Shall we dance? The importance of staff-student relationships to undergraduate dissertation preparation. Active Learning in Higher Education, 12(2), 91-100.

DiPardo, A. \& Freedman, S. W. (1988). Peer response groups in the writing classroom: Theoretic foundations and new directions. Review of educational research, 58(2), 119-149.

Dysthe, O. (2012). Multivoiced classrooms in higher education academic writing. En M. Castelló \& C. Donahue (Eds.), University writing: Selves and Texts in Academic Societies (pp. 201-216). Londres: Emerald Group.

Dysthe, O., Samara, A. \& Westrheim, K. (2006). Multivoiced supervision of Master's students: A case study of alternative supervision practices in higher education. Studies in Higher education, 31(3), 299-318. 
Gallego, L., Castelló, M. \& Badia, A. (2015). Faculty feelings as writers: Relationship with writing genres, perceived competences, and values associated to writing. Higher Education, 1-16.

Giroux, H. A. (1990). Los profesores como intelectuales: Hacia una pedagogía crítica del aprendizaje. Barcelona: Paidós.

Greenbank, P. \& Penketh, C. (2009). Student autonomy and reflections on researching and writing the undergraduate dissertation. Journal of Further and Higher Education, 33(4), 463-472.

Harland, J., Pitt, S. \& Saunders, V. (2005) Factors affecting student choice of the undergraduate research project: Staff and student perceptions. Bioscience Education, 5 [en línea]. Disponible en: http://www.bioscience.heacademy/journal/vol5/beej-5-3.pdf

Harrison, M. E. \& Whalley, W. B. (2006). Combining student independent learning and peer advice to improve the quality of undergraduate dissertations. Planet, $16,15-18$.

Harrison, M. E. \& Whalley, W. B. (2008). Undertaking a dissertation from start to finish: The process and product. Journal of Geography in Higher Education, 32(3), 401-418.

Heinze, A. \& Heinze, B. (2009). Blended e-learning skeleton of conversation: Improving formative assessment in undergraduate dissertation supervision. British Journal of Educational Technology, 40(2), 294-305.

Heylings, D. J. A. \& Tariq, V. N. (2001). Reflection and feedback on learning: A strategy for undergraduate research project work. Assessment \& evaluation in Higher education, 26(2), 153-164.

Hyland, K. (2005). Stance and engagement: A model of interaction in academic discourse. Discourse Studies, 7, 173-192. DOI: 10.1177/1461445605050365.

Inman, A. G. \& Silverstein, M. E. (2003). Dissertation support group: To dissertate or not is the question, Journal of College Student Psychotherapy, 17(3), 59-69.

Kruse, O. (2003). Getting started: Academic writing in the first year. En B. Lennart, G. Bräuer, L. Rienecker \& P. Stray Jörgensen (Eds.), Teaching academic writing in european higher education (pp. 19-28). Netherlands: Kluwer Academic Publishers.

Latorre, A. (2003). Investigación acción. Barcelona: Graó.

Lea, M. R. \& Stierer, B. (2000). Student writing in higher education: New contexts. Buckingham, UK: SRHE \& Open University Press. 
López-Cózar, C., Priede, T. \& Benito, S. (2013). Análisis de la expresión escrita en las Universidades de Madrid a través de la asignatura Trabajo Fin de Grado en los estudios de ADE. Revista de Docencia Universitaria. REDU, 11(3), 279-299.

Martí, M. L., Marzal, C. C. \& Medina, R. P. (2013). Gestión y evaluación del trabajo final de carrera en Administración y Dirección de Empresas. Revista de Formación e Innovación Educativa Universitaria, 6(3), 196-205.

Moyano, E. (2004). La escritura académica: Una tarea interdisciplinaria a lo largo de la currícula universitaria. Revista Texturas, 4(4), 109-120.

Orsmond, P., Merry, S. \& Reiling, K. (2004). Undergraduate project work: Can directed tutor support enhance skill development? Assessment and Evaluation in Higher Education, 29(5), 625-641.

Pañellas, M., Alguacil, M., Boqué, M. C. \& Rosich, C. (2013). Diseño de un módulo integrador del Trabajo Final de Grado y el Prácticum en la formación de maestros. Valoración de las escuelas. Cultura y Educación, 25(3), 259-272.

Rekalde, I. (2011). ¿Cómo afrontar el trabajo de fin de grado? Un problema o una oportunidad para culminar con el desarrollo de las competencias. Revista Complutense de Educación, 22(2), 179-193.

Rinck, F. \& Boch, F. (2012). Enunciative strategies and expertise levels in academic writing: How do writers manage point of view and sources? En M. Castelló \& C. Donahue (Eds.), University writing: Selves and Texts in Academic Societies (pp. 111-128). Londres: Emerald Group.

Russell, D. R. \& Cortes, V. (2012). Academic and scientific texts: The same or different communities? En M. Castelló \& C. Donahue (Eds), University writing: Selves and texts in academic societies (pp. 177-200). Londres: Emerald Group.

Ryder, J. (2004). What can students learn from final year research projects? Bioscience Education, 4. DOI: 10.3108/beej.2004.04000003.

Sánchez Fernández, P. (2013). Trabajo Fin de Grado en Administración y Dirección de Empresas (ADE): De la teoría a la experiencia de la Facultad de CC. Empresariales y Turismo del campus de Ourense (Universidad de Vigo). Revista de Docencia Universitaria, 11(3), 461-481.

Schön, D. (2002). From technical rationality to reflection-in-action. Supporting lifelong learning, 1, 40-61.

Seymour, E., Hunter, A. B., Laursen, S. L. \& DeAntoni, T. (2004). Establishing the benefits of research experiences for undergraduates in the sciences: First findings from a three-year study. Science Education, 88(4), 493-534. 
Solé, I. \& Miras, M. (2007). La elaboración del conocimiento científico y académico. En M. Castelló (Coord.), Escribir y comunicarse en contextos científicos y académicos. Conocimientos y estrategias (pp. 83-112). Barcelona. Graó.

Swales, J. (2004). Research genres. Exploration and applications. Cambridge: Cambridge University Press.

Todd, M., Bannister, P. \& Clegg, S. (2004). Independent inquiry and the undergraduate dissertation: Perceptions and experiences of final-year social science students. Assessment \& Evaluation in Higher Education, 29(3), 335-355.

Vargas Franco, A. (2005). Escribir en la Universidad: Reflexiones sobre el proceso de composición escrita de textos académicos. Lenguaje, 33, 97-125.

Vilardell, I. (2010). Experiencia sobre el trabajo de fin de Grado en Administración y Dirección de Empresas. Revista de Educación en Contabilidad, Finanzas y Administración de Empresas, 1(1), 101-122.

Zeichner, K. (1993). El maestro como profesional reflexivo. Cuadernos de pedagogía, 220, 44-49.

Zeichner, K. \& Liston, D. (2014). Reflective teaching: An introduction. Nueva York: Routledge.

\section{NOTAS}

${ }^{1}$ La denominación oficial adoptada en España es la de Trabajo Final de Grado (TFG); sin embargo en este trabajo utilizaremos la de Tesis Final de Grado dada su mayor amplitud y similitud con denominaciones similares utilizadas en otros contextos, como: 'Tesis de grado', Undergraduate dissertation, Undergraduate Thesis, Undergraduate Research Project, Undergraduate Final Year Project, o Final Year Research Project.

2 Como es conocido, la normativa APA recoge un conjunto de estándares elaborados por la American Psychological Association con la finalidad de unificar la forma de presentación de trabajos escritos a nivel internacional, diseñados especialmente para artículos y otros documentos de investigación. Ver http://www.apastyle.org/

3 Nos referimos a los recursos que Hyland (2005), a partir del análisis de los recursos presentes en textos científicos publicados en diferentes disciplinas, denominó hedges y boosters.

4 Para más información y acceso a los materiales de escritura, se puede visitar la página $W e b$ del grupo Estrategias de lectura y escritura académica Grupo de investigación SINTE-Lest http://sintelest.wordpress.com/recursos/ 


\section{ANEXO}

\section{Encuesta inicial y final}

\begin{tabular}{|c|c|}
\hline ENCUESTA INICIAL & ENCUESTA FINAL \\
\hline $\begin{array}{l}\text { ¿Cuál crees que es el sentido de } \\
\text { escribir un artículo de investigación en } \\
\text { el contexto de la TFG? } \\
\text { ¿Cómo te sientes al escribir un artículo } \\
\text { de investigación (al principio, durante } \\
\text { la escritura, al final...). ¿Te apetece? } \\
\text { ¿Por qué? } \\
\text { ¿Crees que es fácil o difícil? ¿Por qué? } \\
\text { ¿Qué problemas anticipas? }\end{array}$ & $\begin{array}{l}\text { ¿Cuál crees que es el sentido de escribir un artículo } \\
\text { de investigación en el contexto de la TFG? ¿De qué } \\
\text { te ha servido a ti? } \\
\text { ¿Cómo te has sentido al escribir un artículo de } \\
\text { investigación (al principio, durante la escritura, al } \\
\text { final...)? ¿Te ha gustado? ¿O te has sentido a } \\
\text { disgusto por algún motivo? } \\
\text { ¿Ha sido fácil o difícil? ¿Por qué? } \\
\text { ¿Qué dirías que has aprendido en la TFG? } \\
\text { ¿Cuál es tu valoración general del proceso de ayuda } \\
\text { recibido a lo largo del proceso? } \\
\text { ¿Quién y qué te ha ayudado más? } \\
\text { ¿Cómo valoras la retroalimentación que has recibido } \\
\text { del compañero? ¿Y del profesor? } \\
\text { ¿Cómo valoras tu rol de revisor de los textos de tu } \\
\text { compañero? }\end{array}$ \\
\hline
\end{tabular}

